\title{
Distance between Histograms with Shuffled Cost Matrix
}

\author{
Sung-Hyuk Cha \\ Department of Computer Science, Pace University \\ 861 Bedford rd, Pleasantville, NY 10570 \\ schalpace.edu
}

\begin{abstract}
Comparing two distributions is of fundamental importance to statistics and pattern recognition. The earth mover's distance (EMD) has been considered an excellent distance measure between two distributions. It is defined to minimize the cost using a given cost matrix and formulated as the transportation problem which is a hard optimization problem. There are three special type cost matrices where efficient algorithms are known: nominal, ordinal, and modulo. Here the problem of identifying whether a given cost matrix has the shuffled ordinal property is considered and if so, the linear time complexity algorithm can be applied to compute the $E M D$ efficiently.
\end{abstract}

\section{Introduction}

Comparing two distributions plays important role in pattern classification, clustering, and statistics problems [1]. Among numerous distance or similarity measures, the Earth Mover's Distance, or EMD in short, has received great attention in computer vision and pattern recognition. It is the minimal amount of work that must be performed to transform one distribution into the other by moving distribution mass [2]. The similar concept was called the Mallows distance in Statistics earlier [3].

Let $X$ be a set of $n$ elements whose possible values are finite. A histogram $H(X)$ of a set $X$ represents the frequency of each value in $N$, an ordered list of bin names. Let $b$ be the number of bins. The frequency value of the $i$ th bin is denoted as $H_{i}(X)$. For examples in Figure 1 (a), $n=10, b=4, N=$ [A C G T], $N_{4}=\mathrm{T}, H_{2}(S)=3$, and $H_{1}(T)=$ 2, etc. $H(S)$ and $H(T)$ in Figure 1 (a) shall be used as illustrative examples throughout the rest of this paper.

Computing the EMD between two histograms can be formulated as a transportation problem which is a special linear programming problem to distribute any commodity from any group of supply centers, called sources, to any group of receiving centers, called destinations, in such a way as to minimize the total distribution cost [4]. In general, source $i=(1,2, \ldots, b)$ has a supply of $H_{i}(S)$ units to distribute to the destinations, and destination $j=(1,2, \ldots, b)$ has a demand for $H_{i}(T)$ units to be received from the sources. Let $Z$ be the total distribution cost. Let $x_{i, j}$ and $c_{i, j}$ denote the number of units to be distributed and its cost from a source $i$ to a destination $j$. Then the linear programming formulation of the EMD becomes 


$$
\begin{array}{ccc}
\text { Minimize } & Z=\sum_{i=1}^{b} \sum_{j=1}^{b} c_{i j} x_{i j} \\
\text { subject to } & \sum_{j=1}^{b} x_{i j}=H_{i}(S), \quad \text { for } i=1,2, \ldots, b \\
& \sum_{i=1}^{b} x_{i j}=H_{j}(T), \quad \text { for } j=1,2, \ldots, b \\
\text { and } & x_{i j} \geq 0, \quad \text { for all } i \text { and } j \\
C_{i, j}= & {\left[\begin{array}{ccccc}
A & c_{A, A} & c_{C, A} & c_{G, A} & c_{T, A} \\
C & c_{C, A} & c_{C, C} & c_{G, C} & c_{T, C} \\
G & c_{G, A} & c_{G, C} & c_{G, G} & c_{T, G} \\
T & c_{T, A} & c_{T, C} & c_{T, G} & c_{T, T} \\
& A & C & G & T
\end{array}\right]}
\end{array}
$$

In order to have any feasible solutions, a necessary and sufficient condition for a transportation problem is $\Sigma_{i=1}^{b} s_{i}=\Sigma_{j=1}^{b} d_{j}$ [4]. Since histograms to be compared are of the same size, $\Sigma_{i=1}^{b} H_{i}(S)=\Sigma_{j=1}^{b} H_{j}(T)=n$, the feasible solutions property is met.

The $E M D$ value between two histograms varies depending on the cost matrix. Figure 1 (b), (c), and (d) show feasible solutions in bipartite graphs with edges as (quantity $\times$ cost) and EMD values between $H(S)$ and $H(T)$ using the following $C_{x}, C_{y}$, and $C_{z}$ cost matrices, respectively.

$$
C_{x}=\left[\begin{array}{llll}
0 & 5 & 1 & 7 \\
5 & 0 & 4 & 6 \\
1 & 4 & 0 & 2 \\
7 & 6 & 2 & 0
\end{array}\right] \quad C_{y}=\left[\begin{array}{llll}
0 & 4 & 5 & 1 \\
4 & 0 & 9 & 3 \\
5 & 9 & 0 & 6 \\
1 & 3 & 6 & 0
\end{array}\right] \quad C_{z}=\left[\begin{array}{llll}
0 & 4 & 2 & 2 \\
4 & 0 & 2 & 2 \\
2 & 2 & 0 & 0 \\
2 & 2 & 0 & 0
\end{array}\right]
$$

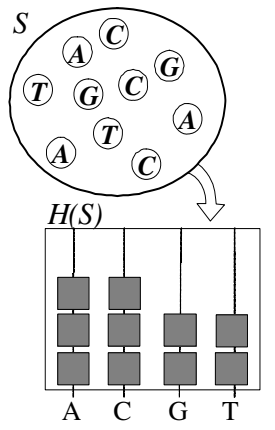

(a)

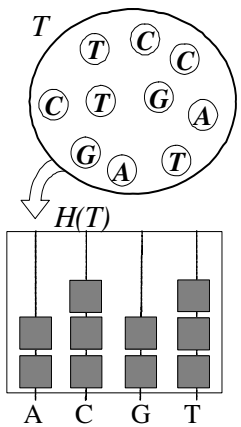

(a)

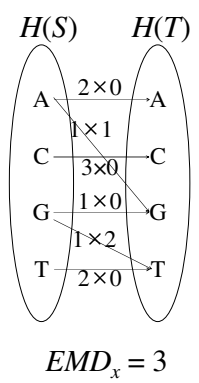

(b)

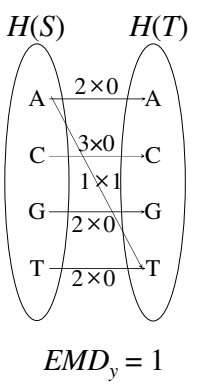

(c)

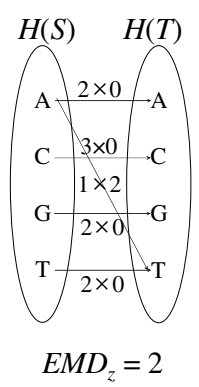

(d)

Fig. 1. Histogram representation and distance between two distributions

The traditional Simplex method [4] can solve the EMD problem and the efficient algorithm takes $O\left(b^{3} \log b\right)$ [2,5]. However, there are special histogram types where the EMD can be computed very fast. The metric was originally called the edit distance [6]. Depending on the measurement types, $\Theta(b), \Theta(b)$, and $O\left(b^{2}\right)$ efficient solutions for computing nominal, ordinal and modulo type histogram edit distances were discovered [6-8]. These special type histograms have corresponding special cost 
matrices. Here the problem of determining whether a cost matrix is one of these special type cost matrices is considered. If so, the efficient algorithm can be applied.

The rest of the paper is organized as follows. In section 2, previously known cost matrices and their algorithms are reviewed and generalized. Section 3 introduces the algorithm to determine whether a given cost matrix has the shuffled-ordinal property. Finally, section 4 concludes this work with open problems.

\section{Previously Known Cost Matrices with Fast Algorithms}

Consider following nominal, ordinal, and modulo cost matrices.

$$
C_{n o m}=\left[\begin{array}{llll}
0 & 1 & 1 & 1 \\
1 & 0 & 1 & 1 \\
1 & 1 & 0 & 1 \\
1 & 1 & 1 & 0
\end{array}\right] \quad C_{\text {ord }}=\left[\begin{array}{llll}
0 & 1 & 2 & 3 \\
1 & 0 & 1 & 2 \\
2 & 1 & 0 & 1 \\
3 & 2 & 1 & 0
\end{array}\right] \quad C_{\text {mod }}=\left[\begin{array}{llll}
0 & 1 & 2 & 1 \\
1 & 0 & 1 & 2 \\
2 & 1 & 0 & 1 \\
1 & 2 & 1 & 0
\end{array}\right]
$$

In a nominal cost matrix, $C_{n o m}$, the diagonal cells have all 0 's and all others have 1 's. Histograms with such a cost matrix were referred to as nominal type histograms [8] and $E M D_{\text {nom }}$ can be computed using the following eqn (3):

$$
E M D_{\text {nom }}(H(S), H(T))=\frac{\sum_{i=1}^{b}\left|H_{i}(S)-H_{i}(T)\right|}{2}
$$

It is nothing but a half of the city block distance and its computational complexity is clearly linear, $\Theta(b)$.

Next, if bin index value is ordered and increases linearly, then these histograms were referred to univariate ordinal type histograms [8]. Let the bin index be denoted as $I$. If an $I=[3,4,5,6]$ for example, then the cost matrix is $C_{\text {ord }}$ and let's denote this special index be $I_{\text {ord }}$. Considering a histogram as a block world, the $E M D_{\text {ord }}$ is the minimum amount of necessary movements to build one source histogram to the other target histogram by moving blocks left or right [6] as illustrated in Figure 2 (a) and can be computed in linear time $\Theta(b)$ using the following formula:

$$
\begin{aligned}
E M D_{\text {ord }}(H(X), H(Y)) & =\sum_{i=1}^{b}\left|\sum_{j=1}^{i} H_{j}(X)-H_{j}(Y)\right| \\
& =\sum_{i=1}^{b-1}\left|\sum_{j=1}^{i} H_{j}(X)-H_{j}(Y)\right|
\end{aligned}
$$

It is calculated by taking the sum of absolute values of prefix sum of difference for each level. Proofs for the correctness and complexity of the algorithm can be found in [6]. Figure 2 (b) shows a couple of feasible solutions for two histograms in Figure 1 (a). The original formula in $[6,8]$ is the eqn (4) but it is equivalent to the eqn (5).

When $I$ values are angular values like north, east, south, and west directions, the first bin and the last bin are neighbors. These kinds of histograms were referred to as angular [7] or modulo type histograms [8] and Figure 2 (c) illustrates the edit distance between two modulo type histograms with $I_{\text {mod }}$ and Figure 2 (d) shows a feasible solution for $E M D_{\text {mod }}$ using the cost matrix $C_{\text {mod }}$. The algorithm and proofs for its correctness and computational time complexity, i.e., $O\left(b^{2}\right)$ can be also found in [8]. 


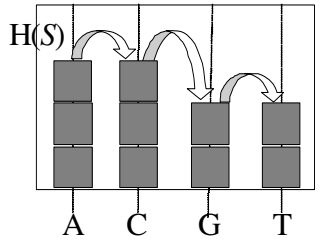

(a) Edit-dist $=3$

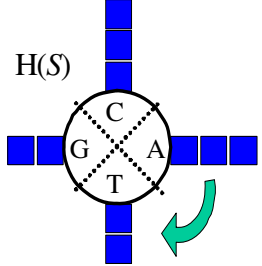

(c) Edit-dist $=1$

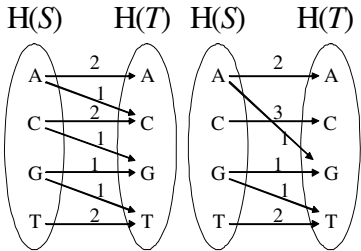

(b) $E M D_{\text {ord }}=3$
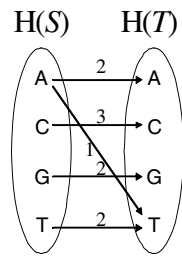

(d) $E M D_{\text {mod }}=1$

Fig. 2. Ordinal and modulo edit distance between histograms in Figure 1

When a constant value $c$ is multiplied to nominal, ordinal, and modulo cost matrices, the new cost matrices also belong to the nominal, ordinal, and modulo type cost matrices, respectively, e.g,

$$
C_{n o m \times 2}=\left[\begin{array}{llll}
0 & 2 & 2 & 2 \\
2 & 0 & 2 & 2 \\
2 & 2 & 0 & 2 \\
2 & 2 & 2 & 0
\end{array}\right] \quad C_{o r d \times 4}=\left[\begin{array}{cccc}
0 & 4 & 8 & 12 \\
4 & 0 & 4 & 8 \\
8 & 4 & 0 & 4 \\
12 & 8 & 4 & 0
\end{array}\right] \quad C_{\bmod \times 3}=\left[\begin{array}{llll}
0 & 3 & 6 & 3 \\
3 & 0 & 3 & 6 \\
6 & 3 & 0 & 3 \\
3 & 6 & 3 & 0
\end{array}\right]
$$

The bin index values in Figure 3 (a) increase by one whereas those in Figure 3 (b) increase by two. Both are ordinal type histograms. Bin values in Figure 3 (c) increase by $2 \pi / b$ and the histogram is a modulo type histogram whose cost matrix is $2 \pi / b \times$ $C_{\text {mod. }}$. If $I_{x}=c \times I_{\text {ord }}$, then a cost matrix $C_{x}=c \times C_{\text {ord }}$

$$
E M D_{x}(H(X), H(Y))=c \times E M D_{\text {ord }}(H(X), H(Y))
$$

The same arguments for nominal and modulo types apply.

So far, bin index values, $I$ in ordinal and modulo histograms increase uniformly by a certain constant value $c$. Consider three histograms in Figure $3(\mathrm{~d} \sim \mathrm{f})$. Their indices and cost matrices are as follows:

$$
\begin{aligned}
& I_{\mathrm{F} 3(\mathrm{~d})}=\left[\begin{array}{llll}
1 & 4 & 5 & 7
\end{array}\right] \quad I_{\mathrm{F} 3(\mathrm{e})}=\left[\begin{array}{llll}
2 & 4 & 4 & 8
\end{array}\right] \quad I_{\mathrm{F} 3(\mathrm{f})}=\left[\begin{array}{llll}
0 & 3 & 6 & 7
\end{array}\right]
\end{aligned}
$$

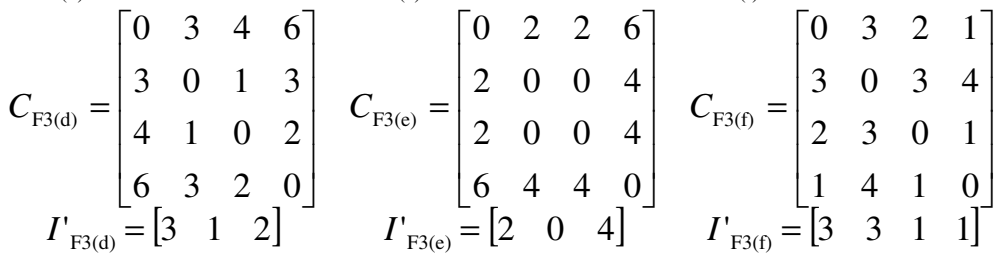




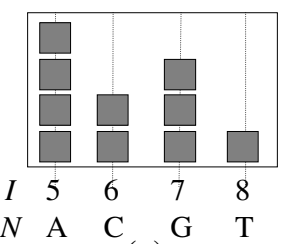

(a)

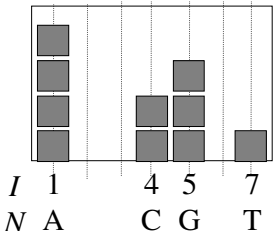

(d)

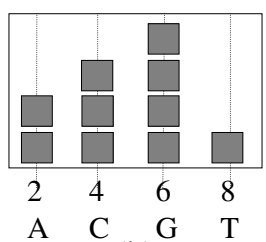

(b)

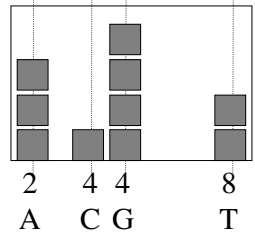

(e)

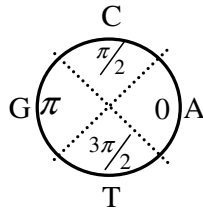

(c)

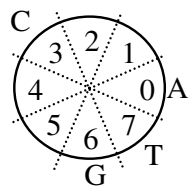

(f)

Fig. 3. Examples of ordinal and modulo type histograms

Albeit these indices do not increase uniformly, the concept of the edit distance [8] can be applied to compute the EMD with these kinds of cost matrices. For example of the ordinal case, the eqn(4) can be generalized to be

$$
E M D_{\text {ord }}(H(X), H(Y))=\sum_{i=1}^{b-1}\left(\left(I_{i+1}-I_{i}\right)\left|\sum_{j=1}^{i} H_{j}(X)-H_{j}(Y)\right|\right)
$$

The term, $\left(I_{i+1}-I_{i}\right)$ is the interval difference $I_{i}$ between two adjacent bin index values. $I_{i}=\left(I_{i+1}-I_{i}\right)=C_{i, i+1}=C_{i+1, i}$. In the $c$-uniform ordinal case, $I_{i+1}-I_{i}=c$ for all bins. Given an $I_{\text {ord }}$, a $C_{\text {ord }}$ can be generated by taking absolute differences. Two different $I_{\text {ord } 1}$ and $I_{\text {ord } 2}$ have the same $C_{\text {ord }}$ as long as $I_{\text {ord } 1}=I_{\text {ord } 2}$. To include the non-uniformly increasing ordinal histograms, the original algorithm in [6] for computing the EMD for ordinal histograms is generalized as follows.

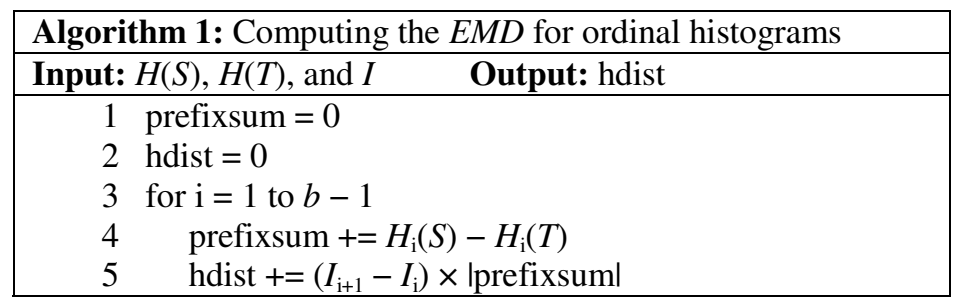

Similarly, the algorithm in [8] for computing the distance between two modulo type histograms can be easily generalized to include the non uniform modulo histograms.

Definition 1: A histogram $H$ is nominal iff .

$$
C_{i, j}=\left\{\begin{array}{l}
0, \text { if } i=j \\
c, \text { otherwise }
\end{array}\right.
$$

Definition 2: A histogram $H$ is ordinal iff the following 3 conditions are met.

1. Elements in $I$ must have scalar numeric values. 
2. $I_{i} \leq I_{j}$ if $i<j$.

3. $C_{i, j}=\left|I_{i}-I_{j}\right|$.

Definition 3: $H$ is modulo iff the following and above $1 \sim 2$ conditions are met.

3. $C_{i, j}=\left\{\begin{array}{ll}\left|I_{i}-I_{j}\right|, & \text { if }\left|I_{i}-I_{j}\right| \leq \beta / 2 \\ \beta-\left|I_{i}-I_{j}\right|, & \text { otherwise }\end{array}\right.$ where $\beta=\sum_{i=1}^{b} I^{\prime}{ }_{i}$

$I_{b}=\left(I_{\mathrm{b}}-I_{1}\right)=C_{b, 1}$ since the last bin and the first bin are neighbors. If all bins in a modulo histogram is crammed in the circular segment s.t. $I_{b} \geq \beta / 2$, it can be realized as an ordinal histogram. Hence, all ordinal histograms are special modulo histograms.

\section{Cost Matrices with the Shuffled Ordinal Property}

Suppose a histogram is given with $C$ and $N$ only without $I$. Determining whether $C$ is nominal is trivial. In this section, a method to determine whether $C$ is ordinal is presented. Let $R^{i}$ be the $i$ th row of $C$, e.g., $R^{1}$ is the first row of $C$.

Definition 4: A cost matrix $C$ is ordinal iff

1. $\quad I\left(=R^{1}\right)$ must satisfy condition 2 in Definition 2 .

2. $C=C^{1}$ where $C^{1}$ is generated by the definition 2 with $R^{1}$ as $I$.

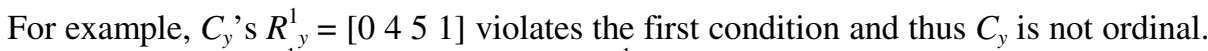
However, $C_{\mathrm{F} 3(\mathrm{~d})}$ 's $R_{\mathrm{F} 3(\mathrm{~d})}^{1}=\left[\begin{array}{llll}0 & 3 & 4 & 6\end{array}\right]$ and $C_{\mathrm{F} 3(\mathrm{~d})}^{1}=C_{\mathrm{F} 3(\mathrm{~d})}$ and thus $C_{\mathrm{F} 3(\mathrm{~d})}$ is ordinal. In other words, $E M D$ with $C_{\mathrm{F} 3(\mathrm{~d})}$ can be solved in linear time using Algorithm 1 whereas $E M D$ with $C_{y}$ may take long time. Here, the concept of shuffling cost matrix to further include more $C$ 's like $C_{y}$ to the linear time complexity cost matrix.

Definition 5: $C_{\pi}$ is a shuffled cost matrix of $C$ if $N_{\pi}$ is a permutation of $N$ and $C_{\pi}$ is generated according to the $N_{\pi}$ order.

For example, some $C_{\pi}$ 's of $C_{\mathrm{F} 4(\mathrm{~d})}$ with $N_{\mathrm{F} 4(\mathrm{~d})}=[\mathrm{A} \mathrm{C} \mathrm{G} \mathrm{T]} \mathrm{include}$

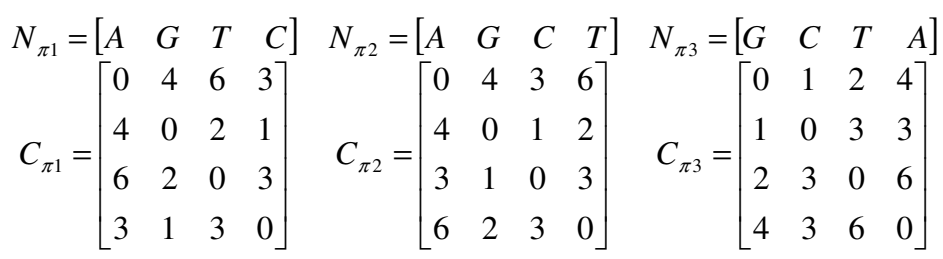

$E M D$ using $C_{\mathrm{F} 4(\mathrm{~d})}=E M D$ using any $C_{\pi}$. While $C_{\mathrm{F} 4(\mathrm{~d})}$ is ordinal, $C_{\pi 1}, C_{\pi 2}$, and $C_{\pi 3}$ are not by definition 4. EMD using any $C_{\pi}$ can be solved in linear time if $N_{\pi}$ is reordered.

Definition 6: A cost matrix $C$ is shuffled-ordinal if $C$ is a $C_{\pi}$ of $C_{o r d}$.

\begin{tabular}{|c|c|}
\hline Algorithm 2: shuffled-ordinality testing for $C$ \\
\hline 1 & $R^{m}=$ any row of $C$ which contains the maximum value in $C$. \\
2 & $R^{s}=$ sort $R^{m}$ in the ascending order \\
3 & $C^{s}{ }_{\text {ord }}=$ the ordinal cost matrix by definition 2 with $R^{s}$ as $I$. \\
4 & $C^{s}=$ the shuffled cost matrix of $C$ according to $R^{s}$ order \\
Definition 7: A cost matrix $C$ is shuffled-ordinal iff $C_{\text {ord }}^{s}=C_{\pi^{s}}$.
\end{tabular}


For earlier example of $C_{x}$, the maximum value 7 is located at first and last rows. If the last row is selected, $R_{x}^{m}=\left[\begin{array}{llll}7 & 6 & 2 & 0\end{array}\right], R_{x}^{s}=\left[\begin{array}{llll}0 & 2 & 6 & 7\end{array}\right]$ and

$$
\left(C_{\text {xord }}^{s}=\left[\begin{array}{cccc}
0 & 2 & 6 & 7 \\
2 & 0 & 4 & 5 \\
6 & 4 & 0 & 1 \\
7 & 5 & 1 & 0
\end{array}\right]\right) \neq\left(C_{x \pi}^{s}=\left[\begin{array}{llll}
0 & 2 & 6 & 7 \\
2 & 0 & 4 & 1 \\
6 & 4 & 0 & 5 \\
7 & 1 & 5 & 0
\end{array}\right]\right)
$$

Hence, $C_{x}$ is not shuffled-ordinal. $C_{y}$ and $C_{z}$ are shuffled-ordinal though. If cost matrices are symmetric, two rows contain the maximum value. If cost matrices such as $C_{z}$ violate the metric separation property, more than two rows may contain the maximum value. Whichever row with the maximum is chosen, the shuffled-ordinal property testing holds. For the example of $C_{y}$, the maximum value 9 appears in the second and third row. Let $R^{s 1}$ and $R^{s 2}$ be the sorted rows using the second and third rows which contain the maximum value, 9 , respectively.

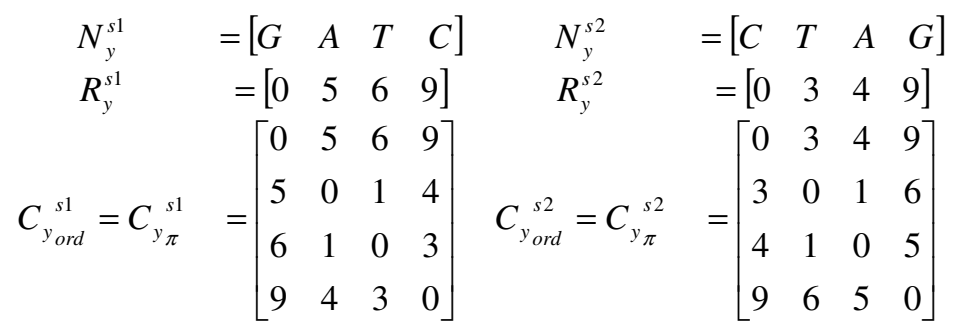

The algorithm 2 finds that $C_{y}$ is shuffled-ordinal using either $R^{s 1}$ or $R^{s 2}$. The new histograms $H^{s 1}$ and $H^{s 2}$ have different orders though. Indeed, $N^{s 1}$ is the reverse order of $N^{s 2}$ and $C^{s 1}$ is the $180^{\circ}$ rotated matrix of $C^{s 2}$. As depicted in Figure $4, H^{s l}$ and $H^{s 2}$ are mirror images of each other and ordinal. Figure 4 assures that

$$
E M D_{y}(H(S), H(T))=E M D_{s 1}\left(H^{s l}(S), H^{s l}(T)\right)=E M D_{s 2}\left(H^{s 2}(S), H^{s 2}(T)\right)=1 .
$$
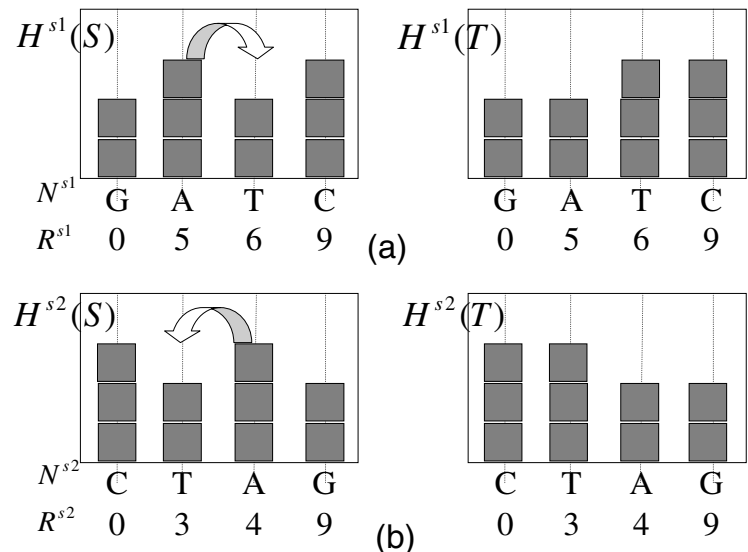

Fig. 4. Edit distances between Shuffled ordinal histograms 
In order to visualize cost matrices, twelve $144 \times 144$ cost matrices are generated randomly as described in Table 1 . Figure 5 shows the cost matrices as images; the darker means the closer to zero and the brighter means the closer to the maximum.

Table 1. Twelve $144 \times 144$ cost matrices with their metric properties

\begin{tabular}{|l|l|c|c|c|c|c|}
\hline$C$ & \multicolumn{1}{|c|}{ Generation method } & $\begin{array}{c}\text { Iden } \\
\text { tity }\end{array}$ & $\begin{array}{c}\text { sepa- } \\
\text { ration }\end{array}$ & $\begin{array}{c}\text { Non } \\
\text {-neg }\end{array}$ & $\begin{array}{c}\text { Sym- } \\
\text { metry }\end{array}$ & $\begin{array}{c}\text { Tri } \\
\text { Ineq }\end{array}$ \\
\hline$C_{1}$ & Ordinal $\left(I_{i+1}-I_{i}=c\right)$. & 1 & 1 & 1 & 1 & 1 \\
\hline$C_{2}$ & Ordinal $\left(I_{i+1} \geq I_{i}\right)$. & 1 & 0 & 1 & 1 & 1 \\
\hline$C_{3}$ & Ordinal $\left(I_{i+1}>I_{i}\right)$. & 1 & 1 & 1 & 1 & 1 \\
\hline$C_{4}$ & Nominal & 1 & 1 & 1 & 1 & 1 \\
\hline$C_{5}$ & Modulo $\left(I_{i+1}-I_{i}=c\right)$. & 1 & 1 & 1 & 1 & 1 \\
\hline$C_{6}$ & Modulo $\left(I_{i+1}>I_{i}\right)$. & 1 & 1 & 1 & 1 & 1 \\
\hline$C_{7}$ & Euclidean $\mathrm{b} / \mathrm{w} 12 \times 12$ D bins. & 1 & 1 & 1 & 1 & 1 \\
\hline$C_{8}$ & Manhattan b/w $12 \times 12$ D bins. & 1 & 1 & 1 & 1 & 1 \\
\hline$C_{9}$ & Symmetric $\left(C_{i, j+1}>C_{i, j}\right)$ for $j \geq i$. & 1 & 1 & 1 & 1 & 0 \\
\hline$C_{10}$ & Symmetric random with 0 & 1 & 0 & 1 & 1 & 0 \\
\hline$C_{11}$ & Symmetric random with Separation & 1 & 1 & 1 & 1 & 0 \\
\hline$C_{12}$ & Totally random & 0 & 0 & 1 & 0 & 0 \\
\hline
\end{tabular}

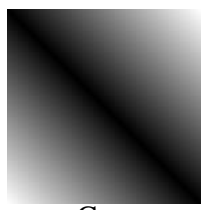

$\mathrm{C}_{1}$

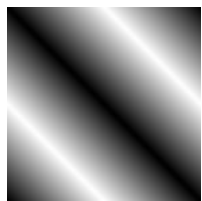

$\mathrm{C}_{5}$

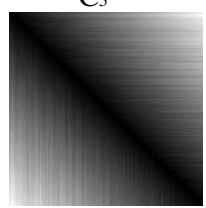

$\mathrm{C}_{9}$

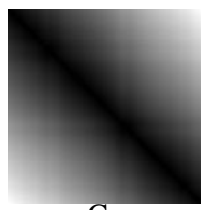

$\mathrm{C}_{2}$

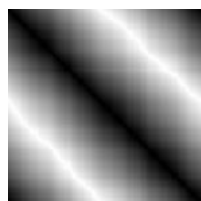

$\mathrm{C}_{6}$

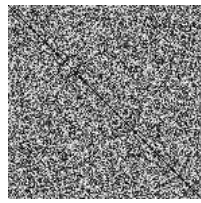

$\mathrm{C}_{10}$

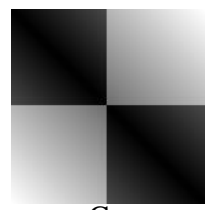

$\mathrm{C}_{3}$

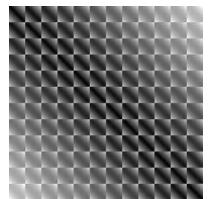

$\mathrm{C}_{7}$

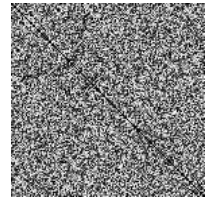

$\mathrm{C}_{11}$

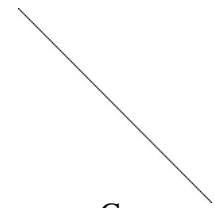

$\mathrm{C}_{4}$

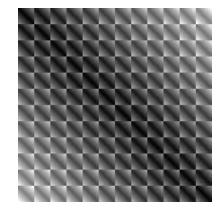

C8

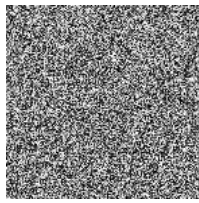

$\mathrm{C}_{12}$

Fig. 5. Cost matrices displayed as images

Randomly shuffling cost matrices in Figure 5, corresponding shuffled cost matrices are displayed in Figure 6. Note that the cost matrix properties in Table 1 are preserved even after shuffling. Identity and symmetry properties are still visible in shuffled cost 

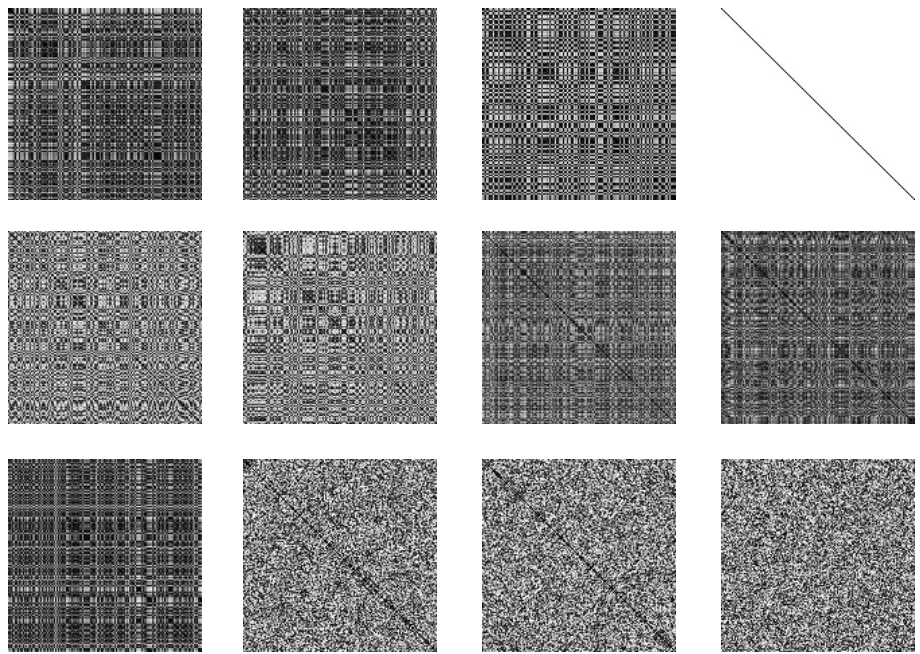

Fig. 6. Randomly shuffled cost matrices of those in Figure 5

matrices $C_{l} \sim C_{11}$. Note that a nominal type cost matrix is shuffling invariant because shuffling does not change the shape of the cost matrix.

Given twelve cost matrices in Figure 6, this section is concerned with the question of which cost matrices are shuffled-ordinal. According the algorithm 2, Figure 7 and Figure 8 shows the $C_{\pi}^{s}$ 's and $C_{\text {ord }}^{s}$ 's for cost matrices in Figure 6, correspondingly. Only $C_{1}, C_{2}$, and $C_{3}$ have $C_{\pi}^{s}=C_{\text {ord }}^{s}$.
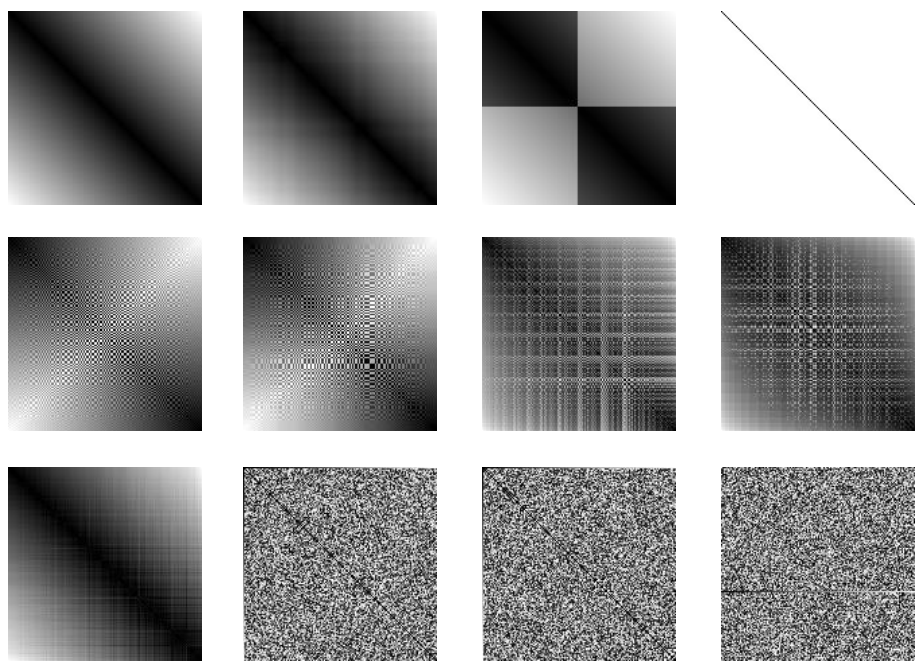

Fig. 7. $C_{\pi}^{s}$, s for cost matrices in Figure 6 

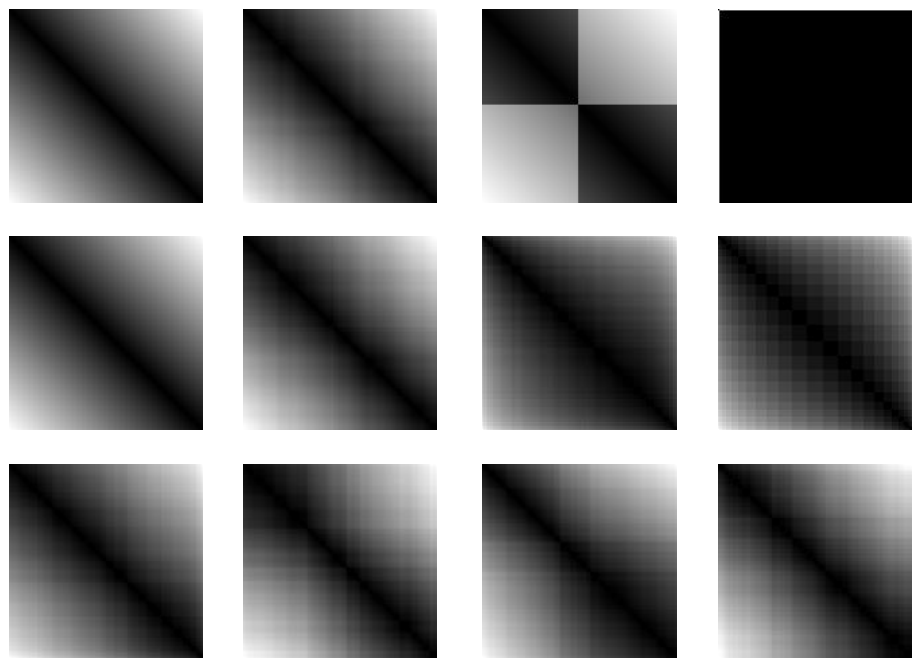

Fig. 8. $C_{\text {ord }}^{s}$ 's for cost matrices in Figure 6

\section{Discussions}

In this paper, three special types of cost matrices are formally defined: nominal, ordinal, and modulo. Computing EMS with these types of cost matrices can be computed very efficiently: $\Theta(b), \Theta(b)$, and $O\left(b^{2}\right)$, respectively. This paper considered the problem of identifying types of cost matrices. A method to identify whether a given cost matrix is shuffled-ordinal was presented. If so, the histograms can be reordered and the linear time complexity algorithm 1 can be applied.

When only the cost matrix is given without types of histogram information, identifying the nominal property is trivial but identifying the shuffled-modulo property is non-trivial. Shuffled-modulo cost matrix can be defined similar to definition 5.

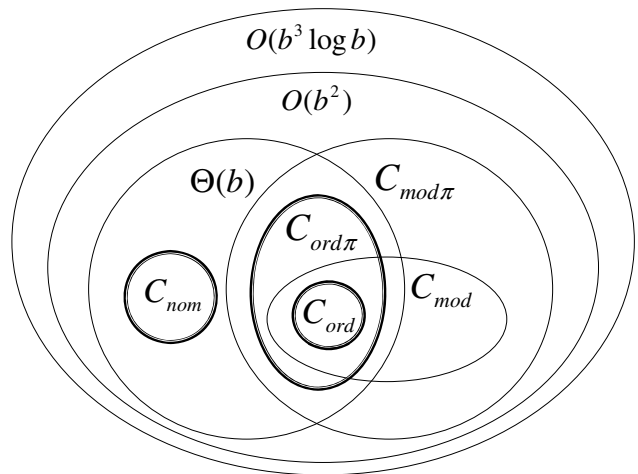

Fig. 9. Sets and their histograms 
Definition 8: A cost matrix $C$ is shuffled-modulo if $C$ is a $C_{\pi}$ of $C_{m o d}$.

Finding the algorithmic definition for $C_{\text {modr }}$ similar to definition 7 is an open problem.

Figure 9 depicts the cost matrices with their best known algorithm complexities. Lower bound algorithms are known only for nominal and ordinal types. Defining new types of cost matrices and finding better algorithms are open problems.

Cost matrices such as BLOSUM [9] and PAM matrices [10] in bio-informatics are often indexed by the alphabetical order of $N$. Indeed, BLOSUM had been the inspiration for this article although it does not have identity, non-negativity properties. Analyzing cost matrices in the real world applications is future works.

\section{References}

1. Duda, R.O., Hart, P.E., Stork, D.G.: Pattern Classification, 2nd edn. Wiley, New York (2000)

2. Rubner, Y., Tomasi, C., Guibas, L.J.: A metric for distributions with applications to image databases. In: Proc. of ICCV, pp. 59-66 (1998)

3. Levina, E., Bickel, P.: The Earth Mover's distance is the Mallows distance: some insights from Statistics. In: Proc. of ICCV, pp. 251-256 (2001)

4. Hiller, F.S., Lieberman, G.J.: Introduction to mathematical programming, 2nd edn. McGraw-Hill, New York (1995)

5. Ahuha, R.K., Magnanti, T.L., Orlin, J.B.: Network Flows. Prentice Hall, Englewood Cliffs (1993)

6. Cha, S.-H.: Fast Image Template and Dictionary Matching Algorithms. In: Chin, R., Pong, T.-C. (eds.) ACCV 1998. LNCS, vol. 1351, pp. 370-377. Springer, Heidelberg (1997)

7. Cha, S.-H., Srihari, S.N.: Distance between Histograms of Angular Measurements and its Application to Handwritten Character Similarity. In: Proceedings of 15th ICPR 2000, September 3-8, 2000, pp. 21-24. IEEE Computer Society, Barcelona (2000)

8. Cha, S.-H., Srihari, S.N.: On Measuring the Distance between Histograms. Journal of Pattern Recognition 35(6), 1355-1370 (2002)

9. Henikoff, S.: Amino Acid Substitution Matrices from Protein Blocks. In: PNAS 1989, pp. 10915-10919 (1992)

10. Dayhoff, M.O., Schwartz, R.M., Orcutt, B.C.: A model of evolutionary change in proteins, matrices for detecting distant relationships. In: Atlas of Protein Sequence and Structure, vol. 5, pp. 345-358. National Biomedical Research Foundation, Washington DC (1978) 